\title{
Modular groups acting on infinite dimensional Teichmüller spaces
}

\author{
Ege Fujikawa
}

\section{Introduction}

For a Riemann surface $R$, the reduced Teichmüller modular group $\operatorname{Mod}^{\#}(R)$ is a group of automorphisms on the reduced Teichmüller space $T^{\#}(R)$. We focus our attention on the proper discontinuity of $\operatorname{Mod}^{\#}(R)$, which is defined as follows.

Definition 1.1. We say that a subgroup $G \subset \operatorname{Mod}^{\#}(R)$ acts properly discontinuously at a point $p \in T^{\#}(R)$ if there exists a neighborhood $U$ of $p$ such that the set $\{\chi \in G \mid \chi(U) \cap U \neq \emptyset\}$ consists of only finitely many elements.

If $R$ is of analytically finite type, $\operatorname{Mod}^{\#}(R)$ and $T^{\#}(R)$ are nothing but the ordinary Teichmüller modular group $\operatorname{Mod}(R)$ and the ordinary Teichmüller space $T(R)$, respectively, and $T^{\#}(R)$ is finite dimensional. In this case, the definition of proper discontinuity is well known and $\operatorname{Mod}^{\#}(R)$ is properly discontinuous at any point in $T^{\#}(R)$. On the other hand, if $R$ is of topologically infinite type, $T^{\#}(R)$ is infinite dimensional and is not locally compact. However the above definition is suitable also in infinite dimensional cases. It is different from the case of finite type that $\operatorname{Mod}^{\#}(R)$ is not necessarily properly discontinuous.

On the basis of the above fact, in the next section, we explain the notions of the limit set and the region of discontinuity for the Teichmüller modular group, which are defined analogously to the theory of Kleinian groups. In section 3, we define the lower and upper bound conditions for Riemann surfaces, which are main subjects in this paper. Further, we give a sufficient condition for the maximal dilatations of quasiconformal maps on Riemann surfaces to be bounded away from one. In section 4 , as an application of the result in section 3, we consider sufficient conditions for a Teichmüller modular group to have a non-empty region of discontinuity. In the last section, we give some examples of Riemann surfaces that satisfy the lower and upper bound conditions by considering normal covering surfaces.

\section{Limit sets and regions of discontinuity}

In this section, we explain the notions of limit sets and regions of discontinuity of Teichmüller modular groups, which were introduced in [5].

(C)2003 American Mathematical Society

1991 Mathematics Subject Classification. Primary 30F60; Secondary 30C62. 
First, we recall the theory of Teichmüller spaces and Teichmüller modular groups (see $[\mathbf{1 0}]$ ). Throughout this paper, we assume that a Riemann surface $R$ is hyperbolic. In other words, it is represented as $\mathbb{H} / \Gamma$ for some torsion-free Fuchsian group $\Gamma$ acting on the upper half-plane $\mathbb{H}$. Furthermore, we assume that $R$ has non-abelian fundamental group. We say that $R$ is of the analytically finite type if it is compact except for finitely many punctures. More precisely, if $R$ is a compact Riemann surface of genus $g$ from which $n$ punctures are removed, then we say that $R$ is of $(g, n)$-type. Further we say that $R$ is of the topologically finite type if it is compact except for finitely many punctures and holes. In other words, $R$ is of the topologically finite type if and only if the Fuchsian model $\Gamma$ of $R$ is finitely generated.

Fix a Riemann surface $R$. For pairs $\left(S_{j}, f_{j}\right)$ of a Riemann surface $S_{j}$ and a quasiconformal map $f_{j}$ of $R$ onto $S_{j}(j=1,2)$, we say that $\left(S_{1}, f_{1}\right)$ and $\left(S_{2}, f_{2}\right)$ are weakly equivalent if there exists a conformal map $h$ of $S_{1}$ onto $S_{2}$ such that $f_{2}^{-1} \circ h \circ f_{1}$ is homotopic to the identity on $R$. The reduced Teichmüller space $T^{\#}(R)$ with the base Riemann surface $R$ is the set of all weakly equivalence classes $[S, f]$ of such pairs $(S, f)$ as above. We say that two quasiconformal automorphisms $h_{1}$ and $h_{2}$ of $R$ are weakly equivalent if they are homotopic on $R$. The reduced Teichmüller modular group $\operatorname{Mod}^{\#}(R)$ is the set of all weakly equivalence classes $[h]$ of quasiconformal automorphisms $h$ of $R$. The reduced Teichmüller space $T^{\#}(R)$ is equipped with the reduced Teichmüller distance $d_{T}(\cdot, \cdot)$ defined by

$$
d_{T}\left(\left[S_{1}, f_{1}\right],\left[S_{2}, f_{2}\right]\right)=\frac{1}{2} \inf _{f_{1}, f_{2}} \log K\left(f_{1} \circ f_{2}^{-1}\right)
$$

where $K(\cdot)$ denotes the maximal dilatation of a quasiconformal map and the infimum is taken over all quasiconformal maps $f_{1}$ and $f_{2}$ determining $\left[S_{1}, f_{1}\right]$ and $\left[S_{2}, f_{2}\right]$, respectively. It is known that, for any quasiconformal map $f$ of $R$ onto $S$, there exists a quasiconformal map having the smallest maximal dilatation in the homotopy class of $f$. This is called an extremal quasiconformal map. The reduced Teichmüller space $T^{\#}(R)$ is a complete metric space with respect to $d_{T}$.

An element $\chi=[h] \in \operatorname{Mod}^{\#}(R)$ induces an automorphism of $T^{\#}(R)$ by

$$
[S, f] \mapsto\left[S, f \circ h^{-1}\right]
$$

which is an isometry with respect to $d_{T}$ and denoted by $\chi_{*}$. Namely, we have a homomorphism of $\operatorname{Mod}^{\#}(R)$ to the automorphism group Aut $\left(T^{\#}(R)\right)$ of $T^{\#}(R)$. With a few exceptional surfaces which do not appear in our present case, the above homomorphism $\operatorname{Mod}^{\#}(R) \rightarrow \operatorname{Aut}\left(T^{\#}(R)\right)$ is faithful. This was first proved in [2]. Another proof was given in $[\mathbf{3}]$. Therefore we identify $\chi_{*}$ with $\chi$ and omit the asterisk hereafter.

We give the definitions of the limit set and the region of discontinuity of a Teichmüller modular group.

Definition 2.1. For a subgroup $G$ of $\operatorname{Mod}^{\#}(R)$, we define $\Omega(G)$ as the set of points $p \in T^{\#}(R)$ where $G$ acts properly discontinuously, and $\Lambda(G)$ as the set of points $p \in T^{\#}(R)$ for which there exists a sequence $\left\{\chi_{n}\right\}$ of distinct elements of $G$ satisfying $\lim _{n \rightarrow \infty} d_{T}\left(\chi_{n}(p), p\right)=0$. We call $\Omega(G)$ the region of discontinuity of $G$, and $\Lambda(G)$ the limit set of $G$. 
Since the action of $\operatorname{Mod}^{\#}(R)$ on $T^{\#}(R)$ is isometric, we see that $\Lambda(G)$ is $G$ invariant and closed (see [5]). We classify the points in $\Lambda(G)$ into three types $\Lambda_{0}(G)$, $\Lambda_{\infty}^{1}(G)$ and $\Lambda_{\infty}^{2}(G)$ according to their stabilizer.

Definition 2.2. In a subgroup $G$ of $\operatorname{Mod}^{\#}(R)$, the stabilizer of a point $p \in$ $T^{\#}(R)$ is defined by $\operatorname{Stab}_{G}(p)=\{\chi \in G \mid \chi(p)=p\}$.

We define $\Lambda_{0}(G)$ as the set of points $p \in \Lambda(G)$ such that there exists a sequence $\left\{\chi_{n}\right\}$ of distinct elements of $G$ satisfying $\lim _{n \rightarrow \infty} d_{T}\left(\chi_{n}(p), p\right)=0$ and $\chi_{n}(p) \neq p$ for all $n$, and $\Lambda_{\infty}(G)$ as the set of points $p \in \Lambda(G)$ such that $\operatorname{Stab}_{G}(p)$ consists of infinitely many elements. Furthermore, we divide $\Lambda_{\infty}(G)$ into two disjoint subsets, $\Lambda_{\infty}^{1}(G)$ and $\Lambda_{\infty}^{2}(G)$. The $\Lambda_{\infty}^{1}(G)$ is the set of points $p \in \Lambda_{\infty}(G)$ such that there exists an element of $\operatorname{Stab}_{G}(p)$ with infinite order, and the $\Lambda_{\infty}^{2}(G)$ is the set of points $p \in \Lambda_{\infty}(G)$ such that all elements of $\operatorname{Stab}_{G}(p)$ are of finite order.

Proposition $2.3([\mathbf{5}])$. Let $G$ be a subgroup of $\operatorname{Mod}^{\#}(R)$. For any point $p$ in $T^{\#}(R)-\Lambda_{0}(G)$, there exists a constant $r>0$ such that the open ball $B(p, r)$ centered at $p$ with radius $r$ is precisely invariant under $\operatorname{Stab}_{G}(p)$. That is, $\chi(B(p, r))=$ $B(p, r)$ for any $\chi \in \operatorname{Stab}_{G}(p)$ and $\chi(B(p, r)) \cap B(p, r)=\emptyset$ for any $\chi \in G-\operatorname{Stab}_{G}(p)$.

Corollary 2.4. $T^{\#}(R)-\Lambda(G)=\Omega(G)$ for any subgroup $G \subset \operatorname{Mod}^{\#}(R)$.

The reduced Teichmüller space $T^{\#}(R)$ is divided into two disjoint subset, the limit set and the region of discontinuity, analogously to the theory of Kleinian groups acting on the Riemann sphere. We expect that they satisfy similar properties to those of limit sets and regions of discontinuity for Kleinian groups. We proved in [5] that $\Lambda(G)-\Lambda_{\infty}^{2}(G)$ does not have an isolated point. In particular, if $\Lambda(G)-$ $\Lambda_{\infty}^{2}(G) \neq \emptyset$, then the limit set $\Lambda(G)$ is an uncountable set.

REMARK 2.5. Similarly, for a subgroup $G$ of the ordinary Teichmüller modular group $\operatorname{Mod}(R)$, we can define $\Lambda(G)$ and $\Omega(G)$ in the ordinary Teichmüller space $T(R)$. However, for a Riemann surface $R$ whose Fuchsian model is of the second kind, we always have $\Omega(\operatorname{Mod}(R))=\emptyset$, since a slight change of the value of a quasiconformal map on the ideal boundary produces a different element of $\operatorname{Mod}(R)$. On the other hand, for a Riemann surface $R$ of analytically finite type, $\Lambda(\operatorname{Mod}(R))=\Lambda\left(\operatorname{Mod}^{\#}(R)\right)=\emptyset$. This is the reason why we consider the reduced Teichmüller modular group $\operatorname{Mod}^{\#}(R)$, not the ordinary Teichmüller modular group $\operatorname{Mod}(R)$, for Riemann surfaces $R$ of infinite type.

\section{The maximal dilatations of quasiconformal maps}

In this section, we give a sufficient condition for the maximal dilatations of quasiconformal maps on Riemann surfaces to be bounded away from one.

The hyperbolic distance on $\mathbb{H}$ is denoted by $d(\cdot, \cdot)$, and the hyperbolic length of a curve $c$ on a Riemann surface $R$ is denoted by $\ell(c)$. For a non-trivial simple closed curve $c$ on $R$ that is not homotopic to a puncture of $R$, we denote the simple closed geodesic that is homotopic to $c$ by $c_{*}$. For a geodesic $L$ on $\mathbb{H}$ and for a quasiconformal automorphism $\tilde{f}$ of $\mathbb{H}$, we denote the geodesic having the same end points as those of $\tilde{f}(L)$ by $\tilde{f}(L)_{*}$.

Definition 3.1. For a constant $M>0$, we define $R_{M}$ to be the set of points $p \in R$ for which there exists a non-trivial simple closed curve $c_{p}$ passing through 
$p$ with $\ell\left(c_{p}\right)<M$. The set $R_{\epsilon}$ is called the $\epsilon$-thin part of $R$ if $\epsilon>0$ is smaller than the Margulis constant. Furthermore, a connected component of the $\epsilon$-thin part corresponding to a puncture is called the cusp neighborhood.

We consider the following conditions in terms of hyperbolic geometry of $R$.

DEFINITION 3.2. We say that $R$ satisfies the lower bound condition if there exists a constant $\epsilon>0$ such that $\epsilon$-thin part of $R$ consists of either cusp neighborhoods or neighborhoods of geodesics which are homotopic to boundary components. We also say that $R$ satisfies the upper bound condition if there exist a constant $M>0$ and a connected component $R_{M}^{*}$ of $R_{M}$ such that the homomorphism of $\pi_{1}\left(R_{M}^{*}\right)$ to $\pi_{1}(R)$ induced by the inclusion map of $R_{M}^{*}$ into $R$ is surjective.

REMARK 3.3. The lower and upper bound conditions are invariant under quasiconformal deformations (see Remark 3.6 and Lemma 3.7).

We state the main result we will prove in this section.

THEOREM 3.4. Let $R$ be a Riemann surface, and $\Gamma$ the Fuchsian model of $R$. Suppose that $R$ satisfies the lower bound condition for a constant $\epsilon>0$ as well as the upper bound condition for a constant $M>0$ and for a connected component $R_{M}^{*}$ of $R_{M}$. Let $\ell>0$ and $C>0$ be constants. Then there exists a constant $A>1$ depending only on $\epsilon, M, \ell$ and $C$ that satisfies the following property: for a quasiconformal automorphism $f$ of $R$, suppose that there exist three distinct axes $L_{i}(i=1,2,3)$ of hyperbolic elements of $\Gamma$ such that

(1) their projections on $R$ are simple closed geodesics $c_{i}(i=1,2,3)$ with $c_{i} \subset R_{M}^{*}$ and $\ell\left(c_{i}\right)<\ell$,

(2) $d\left(z_{1}, L_{2}\right) \leq C$ for some $z_{1} \in L_{1}$,

(3) $\tilde{f}\left(L_{1}\right)_{*}=L_{1}, \quad \tilde{f}\left(L_{2}\right)_{*}=L_{2}, \quad \tilde{f}\left(L_{3}\right)_{*} \neq L_{3}$ for a lift $\tilde{f}$ of $f$ to $\mathbb{H}$. Then $K(f) \geq A$.

Before we prove this theorem, we state an extension of this theorem. First, we define the lower and upper bound conditions for a subdomain of a Riemann surface.

DEFinition 3.5. We say that a subdomain $R^{\prime}$ of $R$ satisfies the lower bound condition if there exists a constant $\epsilon>0$ such that the $R_{\epsilon} \cap R^{\prime}$ consists of either cusp neighborhoods or neighborhoods of geodesics which are homotopic to boundary components. We also say that $R^{\prime}$ satisfies the upper bound condition if there exist a constant $M>0$ and a connected component $U$ of $R_{M} \cap R^{\prime}$ such that the homomorphism of $\pi_{1}(U)$ to $\pi_{1}\left(R^{\prime}\right)$ induced by the inclusion map of $U$ into $R^{\prime}$ is surjective.

Remark 3.6. If we set $R^{\prime}=R$, then Definition 3.5 is nothing but Definition 3.2 for $U=R_{M}^{*}$.

We show that the above conditions are quasiconformally invariant.

LEMMA 3.7. Let $R$ and $S$ be Riemann surfaces, and $f$ a quasiconformal map of $R$ onto $S$. If $R$ has a subdomain satisfying the lower and upper bound conditions, then $S$ also has a subdomain satisfying those conditions.

Proof. Let $\tilde{f}: \mathbb{H} \rightarrow \mathbb{H}$ be a lift of $K$-quasiconformal map $f$. The $\tilde{f}$ can be extended to $\mathbb{H} \cup \hat{\mathbb{R}}$ and the restriction $\tilde{f} \mid \mathbb{R}$ of $\tilde{f}$ to $\mathbb{R}$ is a quasisymmetric function. 
We may assume that $\tilde{f}(\infty)=\infty$. The Douady-Earle extension $\Phi(\tilde{f})$ of $\tilde{f} \mid \mathbb{R}$ to $\mathbb{H}$ is a quasiconformal and bilipschitz map, and the bilipschitz constant $K^{\prime}$ depends only on $K$ (see [1]). The projection $\phi_{f}: R \rightarrow S$ of $\Phi(\tilde{f})$ satisfies $\left(K^{\prime}\right)^{-1} \ell(c) \leq$ $\ell\left(\phi_{f}(c)\right) \leq K^{\prime} \ell(c)$ for an arbitrary curve $c$ on $R$, and $[S, f]=\left[S, \phi_{f}\right]$ in $T^{\#}(R)$.

Let $R^{\prime} \subseteq R$ be a subdomain satisfying the lower and upper bound conditions for positive constants $\epsilon$ and $M$, respectively. Then there exists a connected component $U$ of $R_{M} \cap R^{\prime}$ such that the homomorphism of $\pi_{1}(U)$ to $\pi_{1}\left(R^{\prime}\right)$ induced by the inclusion map of $U$ into $R^{\prime}$ is surjective. For an arbitrary point $p \in \phi_{f}\left(R_{M}\right)$, there exists a non-trivial simple closed curve $c_{p}$ passing through $p$ with $\ell\left(c_{p}\right) \leq K^{\prime} M$. Thus, $\phi_{f}\left(R_{M}\right) \subset S_{K^{\prime} M}$. Therefore, we see that the subdomain $\phi_{f}\left(R^{\prime}\right)$ of $S$ satisfies the upper bound condition for a constant $K^{\prime} M$ and for a connected component of $S_{K^{\prime} M} \cap \phi_{f}\left(R^{\prime}\right)$ containing $\phi_{f}(U)$. Since $R_{\epsilon} \cap R^{\prime}$ consists of either cusp neighborhoods or neighborhoods of geodesics which are homotopic to boundary components, we see that $S_{\left(K^{\prime}\right)^{-1} \epsilon} \cap \phi_{f}\left(R^{\prime}\right)$ also consists of either cusp neighborhoods or neighborhoods of geodesics which are homotopic to boundary components. Hence $\phi_{f}\left(R^{\prime}\right)$ satisfies the lower bound condition for a constant $\left(K^{\prime}\right)^{-1} \epsilon$.

We obtain an extension of Theorem 3.4.

THEOREM 3.8. Let $R$ be a Riemann surface, and $R^{\prime}$ a subdomain of $R$ having non-abelian fundamental group. Suppose that $R^{\prime}$ satisfies the lower bound condition for a constant $\epsilon>0$ as well as the upper bound condition for a constant $M>0$ and for a connected component $U$ of $R_{M} \cap R^{\prime}$. Let $\iota_{*}$ be the homomorphism of $\pi_{1}\left(R^{\prime}\right)$ to $\pi_{1}(R)$ that is induced by the inclusion map $\iota$ of $R^{\prime}$ into $R$, and set $\Gamma^{\prime}=\operatorname{Im} \iota_{*}$ which we regard as a subgroup of the Fuchsian model $\Gamma$ of $R$. Further, let $\ell>0$ and $C>0$ be constants. Then there exists a constant $A>1$ depending only on $\epsilon, M, \ell$ and $C$ that satisfies the following property: for a quasiconformal automorphism $f$ of $R$, suppose that there exist three distinct axes $L_{i}(i=1,2,3)$ of hyperbolic elements of $\Gamma^{\prime}$ such that

(1) their projections on $R$ are simple closed geodesics $c_{i}(i=1,2,3)$ with $c_{i} \subset U$ and $\ell\left(c_{i}\right)<\ell$,

(2) $d\left(z_{1}, L_{2}\right) \leq C$ for some $z_{1} \in L_{1}$,

(3) $\tilde{f}\left(L_{1}\right)_{*}=L_{1}, \tilde{f}\left(L_{2}\right)_{*}=L_{2}, \quad \tilde{f}\left(L_{3}\right)_{*} \neq L_{3} \quad$ for a lift $\tilde{f}$ of $f$ to $\mathbb{H}$. Then $K(f) \geq A$ on $R$.

Remark 3.9. If we set $R^{\prime}=R$, then Theorem 3.8 is nothing but Theorem 3.4.

For proofs of the theorems, we note the following fact on the definition of $R_{M}$.

Proposition $3.10([\mathbf{7}])$. For a constant $M>0$, let $R_{M}^{*}$ be a connected component of $R_{M}$. We assume that $R_{M}^{*}-R_{\epsilon}$ is not of $(0,3)$-type for a positive constant $\epsilon(<M)$. Then there exists a constant $M^{\prime} \geq M$ depending only on $\epsilon$ and $M$ such that, for any point $p \in R_{M}^{*}-R_{\epsilon}$, there exists a non-trivial simple closed curve $c_{p}$ passing through $p$ such that $\ell\left(c_{p}\right)<M^{\prime}$ and $c_{p}$ is not homotopic to a puncture of $R$.

The following proposition on hyperbolic geometry is a basis of proofs of the theorems.

Proposition 3.11 ([7]). Let $\Gamma$ be a Fuchsian model on $\mathbb{H}$ of a Riemann surface $R$. Assume that $\Gamma$ is non-elementary. Let $M_{0}>0$ and $D_{0}>0$ be constants. Then there exists a constant $A>1$ depending only on $M_{0}$ and $D_{0}$ that satisfies the 
following property: for a quasiconformal automorphism $\tilde{f}$ of $\mathbb{H}$ satisfying $\tilde{f} \circ \Gamma \circ$ $\tilde{f}^{-1}=\Gamma$, suppose that there exist three distinct axes $L_{1}, L_{2}$ and $L_{3}$ of hyperbolic elements of $\Gamma$ such that

(1) the projections of $L_{i}(i=1,2,3)$ on $R$ are simple closed geodesics with lengths less than $M_{0}$,

(2) $d\left(z_{1}, L_{2}\right) \leq D_{0}, d\left(z_{1}, L_{3}\right) \leq D_{0}$ for some $z_{1} \in L_{1}$,

(3) $\tilde{f}\left(L_{1}\right)_{*}=L_{1}, \quad \tilde{f}\left(L_{2}\right)_{*}=L_{2}, \quad \tilde{f}\left(L_{3}\right)_{*} \neq L_{3}$.

Then $K(\tilde{f}) \geq A$.

For readers' convenience, we explain the proof of Proposition 3.11, which is based on the Teichmüller Theorem.

Proof of Proposition 3.11. We may assume that the end points of $L_{1}$ are 0 and $\infty$, and that $z_{1}=\sqrt{-1}$. We may also assume that $K(\tilde{f})<2$. The assumption $d\left(\sqrt{-1}, L_{i}\right) \leq D_{0}(i=2,3)$ implies that there exists a constant $r=r\left(D_{0}\right)>0$ depending only on $D_{0}$ such that the Euclidean distance $d_{E}\left(L_{i}\right)$ of two end points of $L_{i}$ satisfies $d_{E}\left(L_{i}\right)>r$. By applying the following Lemma 3.12 to two axes $L_{1}$ and $L_{i}(i=2,3)$, we see that there exists a constant $\delta>0$ depending only on $r$ and $M_{0}$ (that is, depending only on $M_{0}$ and $D_{0}$ ) such that two end points of $L_{i}$ $(i=2,3)$ are in $\{x \in \mathbb{R}|\delta<| x \mid<1 / \delta\}$.

Since $K(\tilde{f})<2$, the length of the projection of $\tilde{f}\left(L_{3}\right)_{*}$ is less than $2 M_{0}$ by Lemma 3.13. By the assumption, we have $\tilde{f}\left(L_{3}\right)_{*} \neq L_{3}$. Applying Lemma 3.12 to axes $\tilde{f}\left(L_{3}\right)_{*}$ and $L_{3}$, we see that there exists a constant $C>0$ depending only on $r$ and $M_{0}$ such that the inequality $|b-\tilde{f}(b)| \geq C$ holds for at least one end point $b$ of $L_{3}$.

Fixing an end point $a$ of $L_{2}$, we denote the hyperbolic distance on $\mathbb{C}-\{0, a\}$ by $d_{a}(\cdot, \cdot)$. By the above consideration, $\delta<|a|<1 / \delta$ and $\delta<|b|<1 / \delta$. Furthermore, by applying Lemma 3.12 to axes $L_{2}$ and $L_{3}$, we have $|a-b|>C_{0}$ for some constant $C_{0}>0$ depending only on $r$ and $M_{0}$. Since $d_{a}(\cdot, \cdot)$ is compatible with the Euclidean distance on $\mathbb{R}^{2}$ except neighborhoods of $0, a$ and $\infty$, we have $d_{a}(b, \tilde{f}(b)) \geq \log C^{\prime}$ for some constant $C^{\prime}>1$ depending only on $C$. We consider a Möbius transformation $\phi$ satisfying $\phi(0)=0, \phi(a)=1$ and $\phi(\infty)=\infty$. Then $d_{1}(b, \tilde{f}(b)) \geq \log C^{\prime \prime}$, where $C^{\prime \prime}$ is a constant depending only on $C^{\prime}$ and $\delta$ (that is, depending only on $M_{0}$ and $\left.D_{0}\right)$. By applying Lemma 3.14, we see that the assertion follows for $A=\left(C^{\prime \prime}\right)^{2}$.

Lemma $3.12([\mathbf{7}])$. For a constant $M>0$, let $L$ and $L^{\prime}$ be two distinct axes of hyperbolic elements of a Fuchsian group $\Gamma$ whose projections to $\mathbb{H} / \Gamma$ are simple closed geodesics with lengths less than $M$. If $L$ and $L^{\prime}$ are disjoint, then they have a distance greater than $C_{1}>0$ depending only on $M$. If $L$ and $L^{\prime}$ intersect each other, then they make an angle greater than $C_{2}>0$ depending only on $M$.

Lemma 3.13 ([12]). Let $f$ be a quasiconformal map of $R$ onto another Riemann surface, and c a non-trivial simple closed curve on $R$. Then the inequality

$$
K(f)^{-1} \ell\left(c_{*}\right) \leq \ell\left(f(c)_{*}\right) \leq K(f) \ell\left(c_{*}\right)
$$

holds.

LEMma $3.14([\mathbf{9}])$. Let $f$ be a quasiconformal automorphism of $\mathbb{C}$ fixing 0 and 1. Suppose that there exists a point $z_{0} \in \mathbb{C}-\{0,1\}$ such that $d_{1}\left(z_{0}, f\left(z_{0}\right)\right)=\log M$ for some constant $M>1$, where $d_{1}($,$) is the hyperbolic distance on \mathbb{C}-\{0,1\}$. Then $K(f) \geq M^{2}$. 
Using Proposition 3.11, we will prove the theorems. By Remark 3.9, we have only to prove Theorem 3.8. The proof is based on the argument in the proof of the main theorem in $[\mathbf{7}]$.

Proof of TheOREM 3.8. First, we observe a property of $U$, which is a connected component of $R_{M} \cap R^{\prime}$. For an arbitrary point $p$ in $U-R_{\epsilon}$, there exists a non-trivial simple closed curve $c_{p}$ passing through $p$ such that $c_{p}$ is not homotopic to a puncture of $R$ and that $\ell\left(c_{p}\right)<M^{\prime}$, where $M^{\prime}=M^{\prime}(\epsilon, M)$ is a constant in Proposition 3.10 depending only on $\epsilon$ and $M$. Since $c_{p} \subset U$, we have $c_{p *} \subset U$. Since $R^{\prime}$ satisfies the lower bound condition for a constant $\epsilon$, we have $\ell\left(c_{p *}\right)>\epsilon$. Hence there exists a constant $B=B\left(\epsilon, M^{\prime}\right)>0$ depending only on $\epsilon$ and $M^{\prime}$ such that $d\left(p, c_{p *}\right) \leq B$. This implies that, for every point $z$ in $\mathbb{H}$ whose projection to $R$ is in $U-R_{\epsilon}$, there exists an axis $L$ of a hyperbolic element of $\Gamma$ such that $d(z, L) \leq B$ and that the projection of $L$ to $R$ is a simple closed geodesic with length less than $M^{\prime}$.

We fix a point $z_{1} \in L_{1}$, and set $D=2\left(B+M^{\prime}+1\right)$. We shall apply Proposition 3.11 to $M_{0}:=\max \left\{\ell, M^{\prime}\right\}$ and $D_{0}:=\max \{C, 2 D\}$. We consider the following two cases.

Case 1: $d\left(z_{1}, L_{3}\right) \leq 2 D$

By applying Proposition 3.11 to the three axes $L_{i}(i=1,2,3)$ and to the constants $M_{0}(\geq \ell)$ and $D_{0}$, we see that there exists a constant $A>1$ depending only on $M_{0}$ and $D_{0}$ (hence, depending only on $\epsilon, M, \ell$ and $C$ ) such that $K(f) \geq A$, and we have thus proved the theorem.

Case 2: $d\left(z_{1}, L_{3}\right)>2 D$

We take the nearest point $z_{3} \in L_{3}$ from $z_{1}$. Since $c_{i} \subset U(i=1,2,3)$, the projections of $z_{1}$ and $z_{3}$ are in $U$. Since $R^{\prime}$ satisfies the upper bound condition for a connected component $U$ of $R_{M} \cap R^{\prime}$, the homomorphism of $\pi_{1}(U)$ to $\pi_{1}\left(R^{\prime}\right)$ induced by the inclusion map of $U$ into $R^{\prime}$ is surjective. Hence, since $L_{1}$ and $L_{3}$ are axes of elements of $\Gamma^{\prime}$, we can take an oriented smooth curve $\alpha$ from $z_{3}$ to $z_{1}$ so that its projection $\hat{\alpha}$ on $R$ lies in $U$. Further, we can take the curve $\alpha$ so that $\hat{\alpha}$ is in $U-R_{\epsilon}$, since $R_{\epsilon}$ consists of either cusp neighborhoods or neighborhoods of geodesics which are homotopic to boundary components by the lower bound condition.

We take points $z_{4}$ and $z_{5}$ on $\alpha$ so that $z_{3}, z_{4}$ and $z_{5}$ are located in this order with respect to the orientation of $\alpha$ and that they satisfy $d\left(z_{3}, z_{4}\right)=d\left(z_{4}, z_{5}\right)=D$. Since $z_{4}$ and $z_{5}$ are points whose projections to $R$ are in $U-R_{\epsilon}$, it follows from the above observation that there exists an axis $L_{4}$ (resp. $L_{5}$ ) of a hyperbolic element of $\Gamma$ such that $d\left(z_{4}, L_{4}\right) \leq B$ (resp. $d\left(z_{5}, L_{5}\right) \leq B$ ) and the projections of $L_{4}$ and $L_{5}$ to $R$ are simple closed geodesics with lengths less than $M^{\prime}$. Since $d\left(z_{3}, w_{4}\right)=$ $d\left(z_{4}, w_{5}\right)>2\left(B+M^{\prime}\right)$, we see that $L_{3}, L_{4}$ and $L_{5}$ are mutually distinct. We take a point $\zeta_{4} \in L_{4}$ so that $d\left(z_{4}, \zeta_{4}\right) \leq B$.

Suppose that $\tilde{f}\left(L_{4}\right)_{*}=L_{4}$ and $\tilde{f}\left(L_{5}\right)_{*}=L_{5}$. Note that $d\left(\zeta_{4}, L_{5}\right) \leq D+2 B$ and $d\left(\zeta_{4}, L_{3}\right) \leq D+B$. By applying Proposition 3.11 to three axes $L_{4}, L_{5}$ and $L_{3}$ and to the constants $M_{0}$ and $D_{0}(>D+2 B)$, we have $K(f) \geq A$, and have completed the proof.

We consider the case that either $\tilde{f}\left(L_{4}\right)_{*} \neq L_{4}$ or $\tilde{f}\left(L_{5}\right)_{*} \neq L_{5}$ is satisfied. We may assume that $\tilde{f}\left(L_{4}\right)_{*} \neq L_{4}$ because the argument below works for the case that $\tilde{f}\left(L_{5}\right)_{*} \neq L_{5}$.

If $d\left(z_{1}, L_{4}\right) \leq 2 D$, then we apply Proposition 3.11 to three axes $L_{1}, L_{2}$ and $L_{4}$ and to the constants $M_{0}$ and $D_{0}$, and we have $K(f) \geq A$. 
If $d\left(z_{1}, L_{4}\right)>2 D$, then we use the argument in the case 2 . We have $z_{6}, z_{7}$ on $\alpha$ such that $z_{4}, z_{6}$ and $z_{7}$ are located in this order with respect to the orientation of $\alpha$ and $d\left(z_{4}, z_{6}\right)=d\left(z_{6}, z_{7}\right)=D$, and have distinct axes $L_{6}, L_{7}$ of hyperbolic elements as above. Repeating this argument, we get desired three axes to which we can apply Proposition 3.11, since $d\left(z_{1}, L_{2 k}\right) \leq 2 D$ for some $k \in \mathbb{N}$.

\section{Teichmüller modular group of the second kind}

In this section, we consider sufficient conditions for a Teichmüller modular group to have a non-empty region of discontinuity.

Definition 4.1. For a subgroup $G$ of $\operatorname{Mod}^{\#}(R)$, we say that $G$ is of the first kind if $\Omega(G)=\emptyset$, and of the second kind if $\Omega(G) \neq \emptyset$

First we show a sufficient condition for $\operatorname{Mod}^{\#}(R)$ to be of the first kind.

Proposition $4.2([5])$. If $R$ does not satisfy the lower bound condition, then $\operatorname{Mod}^{\#}(R)$ is of the first kind.

Sketch OF The Proof of Proposition 4.2. By the assumption, there exists a sequence $\left\{c_{n *}\right\}$ of simple closed geodesics on $R$ such that $c_{n *}$ are not freely homotopic to boundary components and satisfy $\ell\left(c_{n *}\right) \rightarrow 0(n \rightarrow \infty)$. Let $\left[h_{n}\right]$ be an element of $\operatorname{Mod}^{\#}(R)$ that is the Dehn twist along $c_{n}$ for each $n$. We can take a representative $h_{n}$ so that $\lim _{n \rightarrow \infty} K\left(h_{n}\right)=1$. Hence, for $p_{0}=[R, i d]$, we have $\lim _{n \rightarrow \infty} d_{T}\left(\left[h_{n}\right]\left(p_{0}\right), p_{0}\right)=0$, which means that $p_{0} \in \Lambda\left(\operatorname{Mod}^{\#}(R)\right)$. Let $p=[S, f]$ an arbitrary point in $T^{\#}(R)$. By Lemma 3.13, we have $\ell\left(f\left(c_{n}\right)_{*}\right) \rightarrow 0$. Then we can apply the same argument also to $p$.

Next we consider conditions for $\operatorname{Mod}^{\#}(R)$ to be of the second kind. By using a result in the previous section, we have the following which is a basis of our consideration.

Proposition 4.3. Let $R$ be a Riemann surface, and $R^{\prime}$ a subdomain of $R$ having non-abelian fundamental group and satisfying the lower and upper bound conditions. Let $\left\{f_{n}\right\}$ be a sequence of quasiconformal automorphisms of $R$ satisfying the following three conditions:

(1) $f_{n}$ converge to the identity locally uniformly on $R$,

(2) $\lim _{n \rightarrow \infty} K\left(f_{n}\right)=1$,

(3) $f_{n}(c)$ is homotopic to $c$ for all curves $c$ on $R-R^{\prime}$.

Then $f_{n}$ is homotopic to the identity for sufficiently large $n$.

Proof. Let $\iota_{*}$ be the homomorphism of $\pi_{1}\left(R^{\prime}\right)$ to $\pi_{1}(R)$ that is induced by the inclusion map $\iota$ of $R^{\prime}$ into $R$, and set $\Gamma^{\prime}=\operatorname{Im} \iota_{*}$ which we regard as a subgroup of the Fuchsian model $\Gamma$ of $R$. We take a lift $\tilde{f}_{n}$ of $f_{n}$ so that $\tilde{f}_{n}$ converge to the identity locally uniformly on $\mathbb{H}$. Then the isomorphisms $\chi_{n}: \Gamma \rightarrow \Gamma$ induced by $\tilde{f}_{n}$ converge to the identity. We will prove that $\chi_{n}$ are eventually the identity.

We take positive constants $\epsilon$ and $M$ so that $R^{\prime}$ satisfies the lower and upper bound conditions for $\epsilon$ and $M$, respectively. Then there exists a connected component $U$ of $R_{M} \cap R^{\prime}$ such that the homomorphism of $\pi_{1}(U)$ to $\pi_{1}\left(R^{\prime}\right)$ induced by the inclusion map of $U$ into $R^{\prime}$ is surjective. We can take a non-trivial simple closed curve $c \subset U$ such that $\ell(c)<M$ and that $c$ is not homotopic to a puncture of $R$. 
Then $c_{*} \subset U$. Let $\gamma_{1}$ be a hyperbolic element of $\Gamma^{\prime}$ that represents $c_{*}$. Fixing a hyperbolic element $\gamma\left(\neq \gamma_{1}\right) \in \Gamma^{\prime}$, we set $\gamma_{2}=\gamma \circ \gamma_{1} \circ \gamma^{-1} \in \Gamma^{\prime}$. Since $\chi_{n} \rightarrow i d$ $(n \rightarrow \infty)$ and $\Gamma^{\prime}$ is a discrete group, we have $\chi_{n}\left(\gamma_{1}\right)=\gamma_{1}$ and $\chi_{n}\left(\gamma_{2}\right)=\gamma_{2}$ for sufficiently large $n$.

Suppose to the contrary that $\chi_{n}$ are not eventually the identity. Then, there exists an element $\alpha_{n} \in \Gamma^{\prime}$ such that $\chi_{n}\left(\alpha_{n}\right) \neq \alpha_{n}$ for each $n$. We put $\gamma_{n, 1}=$ $\alpha_{n} \circ \gamma_{1} \circ \alpha_{n}^{-1}$ and $\gamma_{n, 2}=\alpha_{n} \circ \gamma_{2} \circ \alpha_{n}^{-1}$, which are elements of $\Gamma^{\prime}$.

We claim that either $\chi_{n}\left(\gamma_{n, 1}\right) \neq \gamma_{n, 1}$ or $\chi_{n}\left(\gamma_{n, 2}\right) \neq \gamma_{n, 2}$ is satisfied. Indeed, suppose to the contrary that both $\chi_{n}\left(\gamma_{n, 1}\right)=\gamma_{n, 1}$ and $\chi_{n}\left(\gamma_{n, 2}\right)=\gamma_{n, 2}$ are satisfied. Then $\beta_{n} \circ \gamma_{j} \circ \beta_{n}^{-1}=\gamma_{j}(j=1,2)$, where $\beta_{n}=\alpha_{n}^{-1} \circ \chi_{n}\left(\alpha_{n}\right)$. Thus, $\beta_{n}$ fixes all fixed points of $\gamma_{1}$ and $\gamma_{2}$. Since $\gamma_{1}$ and $\gamma_{2}$ are non-commutative, the Möbius transformation $\beta_{n}$ fixes four points and it must be the identity map. This contradicts that $\chi_{n}\left(\alpha_{n}\right) \neq \alpha_{n}$.

Thus, without loss of generality, we may assume that $\chi_{n}\left(\gamma_{n, 1}\right) \neq \gamma_{n, 1}$. Let $L_{1}$, $L_{2}$ and $L_{n}$ be axes of $\gamma_{1}, \gamma_{2}$ and $\gamma_{n, 1}$, respectively. Then all the projections of $L_{1}$, $L_{2}$ and $L_{n}$ to $R$ are $c_{*}$, which satisfies $\ell\left(c_{*}\right)<M$. By applying Theorem 3.8 to $\tilde{f}_{n}$ and to the three axes $L_{1}, L_{2}$ and $L_{n}$ for each $n$, we see that there exists a constant $A>1$ depending only on $\epsilon, M$ and $C:=d\left(L_{1}, L_{2}\right)$ (in particular, independent of $n$ ) such that $K\left(f_{n}\right) \geq A$ for all $n$. This contradicts the assumption.

If we set $R^{\prime}=R$ in Proposition 4.3, then we have the following.

COROLlary 4.4. Let $R$ be a Riemann surface satisfying the lower and upper bound conditions, and $\left\{f_{n}\right\}$ a sequence of quasiconformal automorphisms of $R$ satisfying the following two conditions:

(1) $f_{n}$ converge to the identity locally uniformly on $R$,

(2) $\lim _{n \rightarrow \infty} K\left(f_{n}\right)=1$.

Then $f_{n}$ is homotopic to the identity for sufficiently large $n$.

Using the above result, we obtain a candidate for a point of the region of discontinuity.

THEOREM 4.5. Let $R$ be a Riemann surface satisfying the lower and upper bound conditions, and $G$ a subgroup of $\operatorname{Mod}^{\#}(R)$ satisfying the following: there exist two compact subsets $C_{1}$ and $C_{2}$ on $R$ such that, for every $\left[g_{0}\right] \in G$, there is a conformal automorphism $f$ of $R$ satisfying $f \circ g\left(C_{1}\right) \cap C_{2} \neq \emptyset$ for an extremal quasiconformal map $g$ in $\left[g_{0}\right]$. Then $p_{0}=[R, i d] \notin \Lambda_{0}(G)$.

Proof. Let $\left\{\left[g_{n}\right]\right\}$ be a sequence of distinct elements of $G$. We assume that $g_{n}$ is an extremal quasiconformal map in the homotopy class $\left[g_{n}\right]$. Suppose that $\left[g_{n}\right]\left(p_{0}\right) \rightarrow p_{0}(n \rightarrow \infty)$. Then $K\left(g_{n}\right) \rightarrow 1$. We will show that $\left[g_{n}\right]\left(p_{0}\right)=p_{0}$ for sufficiently large $n$. By the assumption, for each $g_{n}$, there exists a conformal automorphism $f_{n}$ of $R$ such that $f_{n} \circ g_{n}\left(C_{1}\right) \cap C_{2} \neq \emptyset$. Then we see that a subsequence of $\left\{f_{n} \circ g_{n}\right\}$ converges to a quasiconformal automorphism of $R$ locally uniformly on $R$. Since $K\left(f_{n} \circ g_{n}\right)=K\left(g_{n}\right) \rightarrow 1$, we may assume that $f_{n} \circ g_{n}$ converge to a conformal automorphism $h$ of $R$. Since $f_{n} \circ g_{n} \circ h^{-1}$ converge to the identity and since $K\left(f_{n} \circ g_{n} \circ h^{-1}\right)=K\left(g_{n}\right) \rightarrow 1$, Corollary 4.4 says that $\left[f_{n} \circ g_{n} \circ h^{-1}\right]=[i d]$ for sufficiently large $n$. Then $\left[g_{n}\right]=\left[f_{n}^{-1} \circ h\right]$ for sufficiently large $n$. Since $f_{n}^{-1} \circ h$ is a conformal automorphism of $R$, we have $\left[g_{n}\right]\left(p_{0}\right)=\left[f_{n}^{-1} \circ h\right]\left(p_{0}\right)=p_{0}$ for sufficiently large $n$. 
We show an example of a Riemann surface satisfying the assumption in Theorem 4.5 .

EXAMPLE 4.6. Let $\hat{R}$ be a topologically finite Riemann surface. For a normal covering surface $R$ of $\hat{R}$ which is not a universal cover and for $G=\operatorname{Mod}^{\#}(R)$, the assumptions of Theorem 4.5 are satisfied.

Indeed, $R$ satisfies the lower and upper bound conditions, which will be proved in Theorem 5.1. Since $\hat{R}$ is a topologically finite Riemann surface, $\hat{R}_{0}=C(\hat{R})-\hat{R}_{\epsilon}$ is compact. Here $C(\hat{R})$ is the convex core of $\hat{R}$ and $\hat{R}_{\epsilon}$ is the $\epsilon$-thin part of $\hat{R}$. Let $\Gamma$ be the covering transformation group for a normal covering surface $R$ of $\hat{R}$, and $C$ a compact subset of $R$ that satisfies $C / \Gamma \supset \hat{R}_{0}$. Then, for any quasiconformal automorphism $g$ of $R$, there exists an element $\gamma \in \Gamma$ such that $g(C) \cap \gamma(C) \neq \emptyset$. Thus $\gamma^{-1} \circ g(C) \cap C \neq \emptyset$, and the assumptions of Theorem 4.5 are satisfied.

COROllary 4.7. Let $R$ be a normal covering surface of a topologically finite Riemann surface such that $R$ is not a universal cover, and $p_{0}=[R, i d] \in T^{\#}(R)$ and $G=\operatorname{Mod}^{\#}(R)$. Then there exists a neighborhood $U$ of $p_{0}$ that is precisely invariant under $\operatorname{Stab}_{G}\left(p_{0}\right)$.

Proof. By Theorem 4.5 and Example 4.6, we have $p_{0} \notin \Lambda_{0}(G)$. Thus, by Proposition 2.3, we have the assertion.

We have a special case of Theorem 4.5.

THEOREM 4.8. Let $R$ be a Riemann surface satisfying the lower and upper bound conditions, and $G$ a subgroup of $\operatorname{Mod}^{\#}(R)$ satisfying the following: there exist compact subsets $C_{1}$ and $C_{2}$ on $R$ such that, for every $\left[g_{0}\right] \in G, g\left(C_{1}\right) \cap C_{2} \neq \emptyset$ for all quasiconformal maps $g$ in $\left[g_{0}\right]$. Then $\Lambda(G)=\emptyset$.

Proof. By Theorem 4.5, we have $p_{0}=[R, i d] \notin \Lambda_{0}(G)$. We set $C=C_{1} \cup C_{2}$. Then $g(C) \cap C \neq \emptyset$ for all quasiconformal maps $g$ in $\left[g_{0}\right] \in G$. Since we assumed that $R$ has non-abelian fundamental group, the group of conformal automorphisms of $R$ acts properly discontinuously ([11, Theorem X.48]). Then there exist only finitely many conformal automorphisms $g$ of $R$ satisfying $g(C) \cap C \neq \emptyset$. Hence, we see that $p_{0} \notin \Lambda_{\infty}(G)$. Therefore, we have proved that $p_{0} \in \Omega(G)$.

Letting $p=[S, f]$ be an arbitrarily point in $T^{\#}(R)$, we set $C^{\prime}=f(C)$. Then $S$ also satisfies the lower and upper bound conditions (see Remark 3.3) and, for every $\left[g_{0}\right] \in G$, a quasiconformal automorphism $g^{\prime}=f \circ g^{-1} \circ f^{-1}$ of $S$ satisfies $g^{\prime}\left(C^{\prime}\right) \cap C^{\prime} \neq \emptyset$ for all $g \in\left[g_{0}\right]$. Thus, by the same consideration as above, we see that $p \in \Omega(G)$.

REMARK 4.9. As we mentioned in the proof of Theorem 4.8, the number of conformal automorphisms of $R$ fixing a compact subset on $R$ is finite. In particular, if a conformal automorphism $f$ of $R$ fixes a compact subset on $R$, then $f$ has finite order. In [4], we proved that, if a conformal automorphism $f$ of $R$ has finite order, then $f$ fixes either a simple closed geodesic, a puncture or a point on $R$. Further, in each case, we obtained a concrete estimate of the order of $f$ in terms of the injectivity radius on $R$.

We have another condition for the limit set to be empty. 
THEOREM 4.10. Let $R$ be a Riemann surface, and $R^{\prime}$ a subdomain of $R$ satisfying the lower and upper bound conditions. Suppose that $R^{\prime}$ and $R-R^{\prime}$ have non-abelian fundamental groups. Let $G$ be a subgroup of $\operatorname{Mod}^{\#}(R)$ such that, for every $[g] \in G, g(c)$ is homotopic to $c$ for all curves $c$ on $R-R^{\prime}$. Then $\Lambda(G)=\emptyset$.

Proof. Since $R-R^{\prime}$ has non-abelian fundamental group, there exists a simple closed geodesic $c_{1}$ on $R-R^{\prime}$ which is not homotopic to a boundary component of $R$. Then there exists a simple closed geodesic $c_{2}\left(\neq c_{1}\right)$ such that $c_{1} \cap c_{2} \neq \emptyset$. Since $g\left(c_{1}\right)$ is homotopic to $c_{1}$ for $[g] \in G$, we have $g\left(c_{1}\right) \cap c_{2} \neq \emptyset$.

First we show that $p_{0}=[R, i d] \in \Omega(G)$. Suppose to the contrary that $p_{0}=$ $[R, i d] \in \Lambda(G)$. Then there exists a sequence $\left\{\left[g_{n}\right]\right\}$ of distinct elements of $G$ such that $\left[g_{n}\right]\left(p_{0}\right) \rightarrow p_{0}(n \rightarrow \infty)$. We assume that $g_{n}$ is an extremal quasiconformal map in the homotopy class $\left[g_{n}\right]$. Then $K\left(g_{n}\right) \rightarrow 1$. Since $g_{n}\left(c_{1}\right) \cap c_{2} \neq \emptyset$, we see that $g_{n}$ converge to a conformal automorphism $h$ of $R$ locally uniformly. Since $f_{n}:=g_{n} \circ h^{-1}$ satisfies the three conditions in Proposition 4.3, the proposition says that $\left[g_{n} \circ h^{-1}\right]=[i d]$ for sufficiently large $n$. Thus $\left[g_{n}\right]=[h]$. This contradicts the assumption that all $\left[g_{n}\right]$ are distinct, and we conclude that $p_{0} \in \Omega(G)$.

A similar argument to the proof of Theorem 4.8 yields that $p \in \Omega(G)$ for any point $p \in T^{\#}(R)$.

As corollaries of Theorem 4.8, we obtain the following two results.

Corollary $4.11([7])$. Let $R$ be a Riemann surface satisfying the lower and upper bound conditions, and $G$ a subgroup of $\operatorname{Mod}^{\#}(R)$ satisfying the following: there exist simple closed geodesics $c_{1}$ and $c_{2}$ on $R$ such that, for every $[g] \in G$, $g\left(c_{1}\right)$ is homotopic to $c_{2}$. Then $\Lambda(G)=\emptyset$.

PROOF. First suppose that $c_{2}$ is not homotopic to a boundary component of $R$. Then there exists a simple closed geodesic $c^{\prime}\left(\neq c_{2}\right)$ on $R$ such that $c_{2} \cap c^{\prime} \neq \emptyset$. Since $g\left(c_{1}\right)$ is homotopic to $c_{2}$ for $[g] \in G$, we have $g\left(c_{1}\right) \cap c^{\prime} \neq \emptyset$. Then Theorem 4.8 concludes $\Lambda(G)=\emptyset$.

Next suppose that $c_{2}$ is homotopic to a boundary component of $R$. We take a pair of pants $P_{i}(i=1,2)$ whose boundary consists of $c_{i}$ and two non-trivial dividing simple closed curves on $R$. Then for every $[g] \in G$, we have $g\left(P_{1}\right) \cap P_{2} \neq \emptyset$. Thus Theorem 4.8 concludes $\Lambda(G)=\emptyset$.

We have a sufficient condition of a Riemann surface for the Teichmüller modular group to act on the Teichmüller space properly discontinuously.

Corollary $4.12([\mathbf{7}])$. Let $R$ be a Riemann surface satisfying the lower and upper bound conditions. Suppose that either the genus, the number of cusps or the number of holes of $R$ is positive finite. Then $\Lambda\left(\operatorname{Mod}^{\#}(R)\right)=\emptyset$.

Proof. First suppose that $R$ is a Riemann surface of genus $g(0<g<\infty)$. Let $l$ be a non-trivial dividing simple closed curve such that one of components of $R-l$ is a Riemann surface $S$ of genus $g$ with only one boundary component. Then for every $\left[g_{0}\right] \in \operatorname{Mod}^{\#}(R), g(\bar{S}) \cap \bar{S} \neq \emptyset$ for all quasiconformal maps $g$ in $\left[g_{0}\right]$. By Theorem 4.8, we have the assertion.

Next suppose that the number of cusps of $R$ is $m(0<m<\infty)$. Let $S$ be a subdomain on $R$ whose boundary consists of $m$ cusps and two non-trivial dividing simple closed curves on $R$. Let $V$ be the union of cusp neighborhoods of $S$. Then 
for every $\left[g_{0}\right] \in \operatorname{Mod}^{\#}(R), g(\bar{S}-V) \cap(\bar{S}-V) \neq \emptyset$ for all quasiconformal maps $g$ in $\left[g_{0}\right]$. By Theorem 4.8, we have the assertion.

Finally suppose that the number of holes of $R$ is $n(0<n<\infty)$. Let $c_{i}$ $(i=1, \cdots, n)$ are simple closed geodesics which are homotopic to the borders. Let $S$ be a subdomain on $R$ whose boundary consists of the $c_{i}(i=1, \cdots, n)$ and two non-trivial dividing simple closed curves on $R$. Then for every $\left[g_{0}\right] \in \operatorname{Mod}^{\#}(R)$, $g(\bar{S}) \cap \bar{S} \neq \emptyset$ for all quasiconformal maps $g$ in $\left[g_{0}\right]$. By Theorem 4.8, we have the assertion.

We consider a condition of Riemann surfaces for corresponding points in $T^{\#}(R)$ to belong the region of discontinuity.

Definition 4.13. The length spectra $\operatorname{LS}(R)$ of a Riemann surface $R$ is the set of all lengths of simple closed geodesics on $R$.

THEOREM 4.14. Let $R$ be a Riemann surface satisfying the lower and upper bound conditions. If a point $p=[S, f] \in T^{\#}(R)$ satisfies the following property $(*)$, then $p$ belongs to $\Omega\left(\operatorname{Mod}^{\#}(R)\right)$.

(*) There exists a simple closed geodesic $c$ on $S$ such that the set

$$
\{x \in \mathbb{R}|| \ell(c)-x \mid<r\} \cap \operatorname{LS}(S)
$$

is a finite subset of $\mathbb{R}$ for some $r>0$. Moreover, for any $\ell>0$ satisfying $|\ell(c)-\ell|<r$, there exist at most finitely many simple closed geodesics on $S$ with lengths $\ell$.

Proof. Suppose to the contrary that $p \in \Lambda\left(\operatorname{Mod}^{\#}(R)\right)$. Then there exist distinct elements $\left[h_{n}\right]$ of $\operatorname{Mod}^{\#}(R)$ such that $\left[h_{n}\right](p) \rightarrow p(n \rightarrow \infty)$. Let $g_{n}$ be an extremal quasiconformal automorphism of $S$ in the homotopy class of $f \circ h_{n}^{-1} \circ f^{-1}$. Then $K\left(g_{n}\right) \rightarrow 1$. We may assume that $K\left(g_{n}\right)<1+r / \ell(c)$ for all $n$. Then, by Lemma 3.13, we see that the geodesic $g_{n}(c)_{*}$ satisfies $\left|\ell(c)-\ell\left(g_{n}(c)_{*}\right)\right|<r$. By the property $(*)$, the set $\left\{g_{n}(c)_{*}\right\}$ consists of finitely many elements. Taking a subsequence of $\left\{g_{n}\right\}$, we may assume that $g_{n}(c)_{*}=g_{1}(c)_{*}$ for all $n$. Then by Corollary 4.11 , we have $p \notin \Lambda\left(\operatorname{Mod}^{\#}(R)\right)$, which is a contradiction.

If $R$ is a compact Riemann surface, then $\operatorname{LS}(R)$ is a discrete subset of $\mathbb{R}$ (see $[8])$. Thus all points $p \in T^{\#}(R)$ satisfy the property $(*)$, and hence $\operatorname{Mod}^{\#}(R)$ acts on $T^{\#}(R)$ properly discontinuously by Theorem 4.14 . The following lemma shows that, if a Riemann surface $R$ of infinite type satisfies the lower bound condition, then there exists a point $p=[S, f] \in T^{\#}(R)$ satisfying the property $(*)$.

LEMma $4.15([5])$. Let $R$ be a Riemann surface satisfying the lower bound condition for a constant $\epsilon>0$, and $c_{0}$ a simple closed geodesic on $R$. Then there exist a positive constant $\alpha<\epsilon$ and a quasiconformal map $f$ of $R$ such that $\ell\left(f\left(c_{0}\right)_{*}\right)<\alpha$ and $\ell\left(f(c)_{*}\right)>\alpha$ for any other simple closed geodesics $c \neq c_{0}$ on $R$.

Theorem 4.14 and Lemma 4.15 yield the following proposition, where the assumption and the conclusion in Corollary 4.12 are weakened.

Proposition 4.16 ([5]). If $R$ satisfies the lower and upper bound conditions, then $\operatorname{Mod}^{\#}(R)$ is of the second kind. 
In connection with Proposition 4.16, we have the following conjecture: "If $R$ satisfies the lower bound condition, then $\operatorname{Mod}^{\#}(R)$ is of the second kind." That is, considering Proposition 4.2, we conjecture that $\operatorname{Mod}^{\#}(R)$ is of the second kind if and only if $R$ satisfies the lower bound condition. We have a partial solution of this conjecture, giving a weaker condition than the upper bound condition.

Proposition $4.17([\mathbf{6}])$. Let $R$ be a Riemann surface satisfying the following two conditions:

(1) $R$ satisfies the lower bound condition.

(2) There exists a constant $M>0$ such that, for any connected component $V$ of $R-R_{M}, V$ is either simply or doubly connected and $R-\bar{V}$ consists of finitely many connected components.

Then $\operatorname{Mod}^{\#}(R)$ is of the second kind.

REMARK 4.18. It is easily seen that, if $R$ satisfies the upper bound condition, then $R$ satisfies the second condition in Proposition 4.17 .

The proof of Proposition 4.17 is based on Theorem 3.8. The examples of Riemann surfaces satisfying the two conditions in this proposition are in Examples 5.6 and 5.7 .

\section{The lower and upper bound conditions}

In the previous section, we showed that the lower and upper bound conditions are sufficient conditions for Teichmüller modular groups to be of the second kind. In this section, we consider some examples of Riemann surfaces satisfying those conditions. Further we give examples of Riemann surfaces such that both the limit sets and the regions of discontinuity of the Teichmüller modular groups are not empty.

THEOREM 5.1. Let $\hat{R}$ be a topologically finite Riemann surface, and $R$ a normal covering surface of $\hat{R}$ which is not a universal cover. Then $R$ satisfies the lower and upper bound conditions.

ProOF. The lower bound condition is clearly satisfied. We will prove that $R$ satisfies the upper bound condition. We set $\hat{R}_{0}=C(\hat{R})-\hat{R}_{\epsilon}$, where $C(\hat{R})$ is the convex core of $\hat{R}$ and $\hat{R}_{\epsilon}$ is the $\epsilon$-thin part of $\hat{R}$. The lift $R_{0}$ of $\hat{R}_{0}$ to $R$ is connected and the homomorphism of $\pi_{1}\left(R_{0}\right)$ to $\pi_{1}(R)$ induced by the inclusion map of $R_{0}$ into $R$ is surjective. We will show that $R_{0} \subset R_{M}$ for some $M>0$. Then the upper bound condition is satisfied for the constant $M$. Since $R$ is a normal covering surface of $\hat{R}$ which is not a universal cover, we can take a simple closed geodesic $\hat{c}_{*}$ on $\hat{R}$ so that the lifts of $\hat{c}_{*}$ to $R$ are closed geodesics. For an arbitrary point $p \in R_{0}$, let $\hat{p} \in \hat{R}_{0}$ be the projection of $p$. We connect $\hat{p}$ and $\hat{c}_{*}$ by the shortest geodesic $\hat{\ell}$. Since $\hat{R}_{0}$ is compact, there exists a constant $M_{1}$ such that the hyperbolic length of $\hat{\ell}$ is less than $M_{1}$ for all $\hat{p} \in \hat{R}_{0}$. Hence there exists a non-trivial simple closed curve $\hat{c}_{p}$ passing through $\hat{p}$ whose hyperbolic length is less than $M=2 M_{1}+M_{2}$, where $M_{2}$ is the hyperbolic length of $\hat{c}_{*}$. By considering the lift $c_{p}$ of $\hat{c}_{p}$ passing through $p$, we conclude that $p \in R_{M}$.

By Proposition 4.16 and Theorem 5.1, the following corollary is obtained. 
COROLlary 5.2. Let $\hat{R}$ be a topologically finite Riemann surface, and $R$ a normal covering surface of $\hat{R}$ which is not a universal cover. Then $\operatorname{Mod}^{\#}(R)$ is of the second kind.

EXAMPLE 5.3. Let $\hat{R}$ be a compact Riemann surface of genus $g \geq 2$, and $R$ a normal covering surface of $\hat{R}$ whose covering transformation group is a cyclic group $\langle\phi\rangle$ generated by a conformal automorphism $\phi$ of $R$. Then $p_{0}=[R, i d] \in T^{\#}(R)$ and $[\phi] \in \operatorname{Mod}^{\#}(R)$ satisfy $[\phi]\left(p_{0}\right)=p_{0}$. Hence $p_{0}$ belongs to $\Lambda_{\infty}^{1}\left(\operatorname{Mod}^{\#}(R)\right)$. On the other hand, $\operatorname{Mod}^{\#}(R)$ is of the second kind by Corollary 5.2. Thus $\Lambda\left(\operatorname{Mod}^{\#}(R)\right) \neq \emptyset$ and $\Omega\left(\operatorname{Mod}^{\#}(R)\right) \neq \emptyset$.

In connection with Theorem 5.1, we see that a Riemann surface inherits the lower and upper bound conditions from its normal covering surface. This is proved by using the result stated in Remark 4.9 .

Proposition 5.4 ([4]). Let $R$ be a Riemann surface and $\tilde{R}$ a normal covering surface of $R$. If $\tilde{R}$ satisfies the lower and upper bound conditions, then $R$ also satisfies these conditions.

From Propositions 4.16 and 5.4, we have a condition of a normal covering surface $\tilde{R}$ for $\operatorname{Mod}^{\#}(R)$ to be of the second kind.

COROLlary 5.5. If $\tilde{R}$ satisfies the lower and upper bound conditions, then both $\operatorname{Mod}^{\#}(\tilde{R})$ and $\operatorname{Mod}^{\#}(R)$ are of the second kind.

If $\tilde{R}$ does not satisfy the upper bound condition, then Proposition 5.4 and Corollary 5.5 are not necessarily true.

ExAmPle 5.6. Let

$$
\tilde{R}=\mathbb{C}-\bigcup_{n=1}^{\infty} \bigcup_{m \in \mathbb{Z}}\left\{\frac{m}{n} \pm n^{2} \sqrt{-1}\right\},
$$

and $R=\tilde{R} /\langle f\rangle$, where $f(z)=z+1$. By a similar consideration to that in $[7$, Example 2], we see that $\tilde{R}$ satisfies the lower bound condition but does not satisfy the upper bound condition. However it satisfies the second condition in Proposition 4.17. Indeed, there exists a constant $M>0$ such that, for any connected component $V$ of $\tilde{R}-\tilde{R}_{M}, V$ is simply connected and $\tilde{R}-\bar{V}$ consists of two connected components. Then $\operatorname{Mod}^{\#}(\tilde{R})$ is of the second kind. On the other hand, $R$ does not satisfy the lower bound condition. Indeed, let $c_{n}$ be the geodesic on $R$ being homotopic to a simple closed curve $\left\{\left(n^{2}+1\right) \sqrt{-1}\right\} /\langle f\rangle$. For a sufficiently small constant $\epsilon>0$, we set $A_{n}=\left\{x+y \sqrt{-1} \in \mathbb{C} \mid n^{2}+\epsilon<y<(n+1)^{2}-\epsilon\right\} /\langle f\rangle$, which is an annulus containing $c_{n}$. By considering the modulus of $A_{n}$, we see that $\ell\left(c_{n}\right) \rightarrow 0(n \rightarrow \infty)$. Hence, by Proposition $4.2, \operatorname{Mod}^{\#}(R)$ is of the first kind.

We have an example that $\tilde{R}$ does not satisfy the upper bound condition but both $\operatorname{Mod}^{\#}(\tilde{R})$ and $\operatorname{Mod}^{\#}(R)$ are of the second kind.

Example 5.7. Let

$$
\tilde{R}=\mathbb{C}-\bigcup_{n=1}^{\infty} \bigcup_{m \in \mathbb{Z}}\left\{\frac{m}{n}+(2 n+1) \sqrt{-1}\right\} .
$$


Although $\tilde{R}$ does not satisfy the upper bound condition (see [7, Example 2]), it satisfies the two conditions in Proposition 4.17 (see [6]). Then $\operatorname{Mod}^{\#}(\tilde{R})$ is of the second kind, that is, $\Omega\left(\operatorname{Mod}^{\#}(\tilde{R})\right) \neq \emptyset$. Further we see that $\Lambda\left(\operatorname{Mod}^{\#}(\tilde{R})\right) \neq \emptyset$. Indeed, set

$$
f_{n}(z)= \begin{cases}x-(y-2 n-2) / n+y \sqrt{-1} & (2 n+1 \leq y<2 n+2) \\ x+(y-2 n) / n+y \sqrt{-1} & (2 n \leq y<2 n+1) \\ x+y \sqrt{-1} & \text { elsewhere. }\end{cases}
$$

Then $f_{n}$ are quasiconformal automorphisms of $\tilde{R}$ satisfying $K\left(f_{n}\right) \rightarrow 1(n \rightarrow \infty)$. Hence $\left[f_{n}\right]\left(p_{0}\right) \rightarrow p_{0}$, where $p_{0}=[\tilde{R}, i d]$. This means that $p_{0} \in \Lambda\left(\operatorname{Mod}^{\#}(\tilde{R})\right)$.

Let $R=\tilde{R} /\langle f\rangle$, where $f(z)=z+1$. Then we see that $R$ satisfies the lower and upper bound conditions (cf. Example 5.6). Thus, by Proposition 4.16, $\operatorname{Mod}^{\#}(R)$ is also of the second kind.

In the last of this section, we propose the following problem: "If $\operatorname{Mod}^{\#}(\tilde{R})$ is of the first kind, then so is $\operatorname{Mod}^{\#}(R)$ ?" It is clear that, if $\tilde{R}$ does not satisfy the lower bound condition, then neither does $R$. Hence, from Proposition 4.2, if $\tilde{R}$ does not satisfy the lower bound condition, then both $\operatorname{Mod}^{\#}(\tilde{R})$ and $\operatorname{Mod}^{\#}(R)$ are of the first kind. Further, if the conjecture, which is stated in the previous section, is true, then this problem is solved affirmatively.

\section{References}

[1] A. Douady and C. J. Earle, Conformally natural extension of homeomorphisms of the circle, Acta Math. 157 (1986) 23-48.

[2] C. J. Earle, F. P. Gardiner and N. Lakic, Teichmüller spaces with asymptotic conformal equivalence, preprint.

[3] A. Epstein, Effectiveness of Teichmüller modular groups, In the tradition of Ahlfors and Bers, Contemporary Math. 256, American Mathematical Society, 2000, 69-74

[4] E. Fujikawa, The order of conformal automorphisms of Riemann surfaces of infinite type, Kodai Math. J. 26 (2003), 16-25. (- supplement, Perspectives of Hyperbolic Spaces, RIMS Kokyuroku 1329 (2003), 62-68.)

[5] E. Fujikawa, Limit sets and regions of discontinuity of Teichmüller modular groups, Proc. Amer. Math. Soc. 132 (2004), 117-126.

[6] E. Fujikawa, Teichmüller modular groups with non-empty regions of discontinuity, preprint.

[7] E. Fujikawa, H. Shiga and M. Taniguchi, On the action of the mapping class group for Riemann surfaces of infinite type, J. Math. Soc. Japan, to appear.

[8] F. P. Gardiner, Teichmüller theory and quadratic differentials, Wiley-Interscience, New York, 1987.

[9] I. Kra, On Teichmüller's theorem on the quasi-invariance of cross ratios, Israel. J. Math., 30 (1978), 152-158.

[10] S. Nag, The Complex Analytic Theory of Teichmüller Spaces, John Wiley \& Sons 1988.

[11] M. Tsuji, Potential Theory in Modern Function Theory, Chelsea, New York, 1959.

[12] S. A. Wolpert, The length spectra as moduli for compact Riemann surfaces, Ann. Math. 109 (1979), 323-351.

Department of Mathematics, Tokyo Institute of Technology, Oh-OKayama MeguroKU TOKYO 152-8551, JAPAN

E-mail address: fujikawa@math.titech.ac.jp 